\title{
Photometric Titration Method to Determine Bromination of Red and Yellow Dyes in Crackers
}

\author{
Jutti Levita $^{1}$, Sandra Megantara ${ }^{1} \&$ Mutakin $^{1}$ \\ ${ }^{1}$ Pharmacochemistry Division, Faculty of Pharmacy, Universitas Padjadjaran \\ Correspondence: Jutti Levita, Jl. Raya Bandung-Sumedang Km 21, Jatinangor, West Java 45363, Indonesia. \\ E-mail: jutti.levita@unpad.ac.id
}

Received: March 21, 2012

Accepted: April 9, $2012 \quad$ Online Published: May 27, 2012

doi:10.5539/ijc.v4n3p80

URL: http://dx.doi.org/10.5539/ijc.v4n3p80

\begin{abstract}
In this work, we determined red and yellow dyes in unregistered crackers sold at Jatinangor, West Java, Indonesia, by using photometric titration method based on the bromination reaction of the dyes. The dyes were extracted using a mixture of $2 \%$ of ammonia in $70 \%$ ethanol continued by preparative thin layer chromatography on GF254 silica gel plate, then they were separated and analyzed by using FTIR- 8400 Shimadzu infrared spectrophotometer. Bromination reaction was measured by using photometric titration method. Thin layer chromatograms showed that the dyes were not either rhodamine B or tartrazine ( $\mathrm{Rf}$ of the red and yellow dyes were 0.714 and 0.652 , respectively, while those of rhodamine B and tartrazine were 0.591 and 0.673). Infrared spectroscopy showed characteristic peaks of the red dye at $3390.6 \mathrm{~cm}^{-1}$ (O-H stretch), $2927.7 \mathrm{~cm}^{-1}$ (C-H stretch of alkyl), $1404.1 \mathrm{~cm}^{-1}$ (O-H bend), $1616.2 \mathrm{~cm}^{-1}$ (C=C stretch), and $1315 \mathrm{~cm}^{-1}$ (C-O stretch). The peaks of yellow dye occured at $3413.8 \mathrm{~cm}^{-1}$, (O-H stretch), $3213.2 \mathrm{~cm}^{-1}$ (C-H stretch of alkene), $2927.7 \mathrm{~cm}^{-1}$ (C-H stretch of alkyl), $1400.2 \mathrm{~cm}^{-1}$ (O-H bend), $1624.0 \mathrm{~cm}^{-1}$ (C=C stretch). Visible spectroscopy showed maximum wavelengths at $551 \mathrm{~nm}$ (red dye) and $432 \mathrm{~nm}$ (yellow dye). Photometric titration based on bromination reaction of the dyes showed a decreasing in absorbance due to the conversion of colored analytes to colorless products. This fact indicated that bromination reaction was occured in the red and yellow dyes molecules via electrophilic addition reaction on its double bond, therefore this method is applicable to be used in routine analysis to control illegal food dyes often found in Indonesian home industry crackers.
\end{abstract}

Keywords: bromination reaction, crackers, illegal food dyes, photometric titration

\section{Introduction}

The change in absorbance of a solution may be used to follow the change in concentration of a radiation-absorbing constituent during a titration. The absorbance is directly proportional to the concentration of the absorbing constituent. The end point of a photometric titration is located by interpreting the changes in the concentration of an absorbing species, and hence in the absorbance of the solution, during titration. Usually the absorbance is plotted against the volume of titrant added to obtain the titration curve. If the titrant is much more concentrated than the solution being titrated, the titration curve will consist of two straight lines (or, in some cases, a straight line and a curve) that intersect at the end point (Figure 1). Volume change is seldom negligible, and straight lines are obtained only if there is correction for dilution (Willard et al., 1988). 


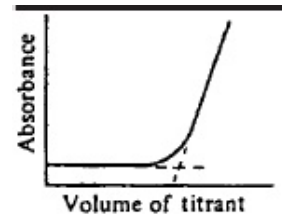

(a)

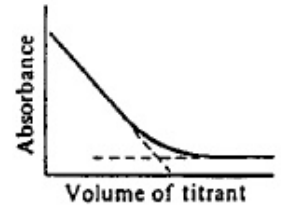

(c)

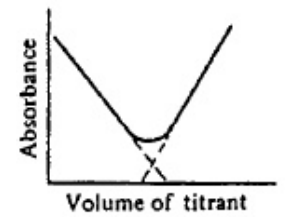

(e)

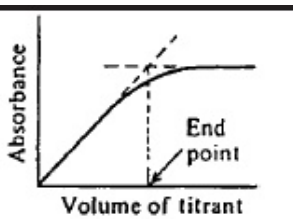

(b)

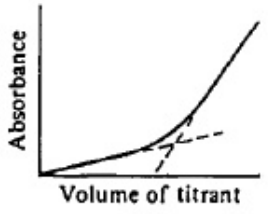

(d)

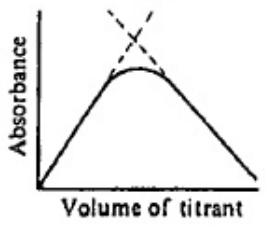

(n)

Figure 1. Possible shapes of photometric titration curves (Willard et al., 1988)

Bromine in solution is reddish-brown. When the free bromine reacts, named bromination, the solution becomes colorless. The reaction can be monitored by simply observing this decrease or disappearance of color. If decolorization of the reddish-brown bromine occurs very soon after the addition of the hydrocarbon, without the need of the light source, the reaction is likely occurring by addition instead of substitution process and the hydrocarbon shows some degree of unsaturation (Solomons, 1980).

Photometric titrations have several distinct advantages over a direct photometric determination. The presence of other absorbing species at the analytical wavelength does not necessarily cause interference, since only the change in absorbance is significant (Willard et al., 1988). Photometric titration can be applied to determine a color analyte, when the analyte is converted to a colorless product by a colored titrant, for example, bromination of red dye (Willard et al., 1988), therefore this method might be useful to determine dyes in colorful crackers (Figure 2), which are favourite food of children and adults in our country, Indonesia.

The simplicity of photometric titration method makes it applicable to be used in routine analysis to control illegal food dyes often found in Indonesian home industry crackers, therefore Indonesian food safety can be achieved as required by Regulation No.722/Menkes/Per/IX/88 of Republic of Indonesia about Food Additives and No.239/Menkes/Per/V/1985 about Prohibited Food Additives.
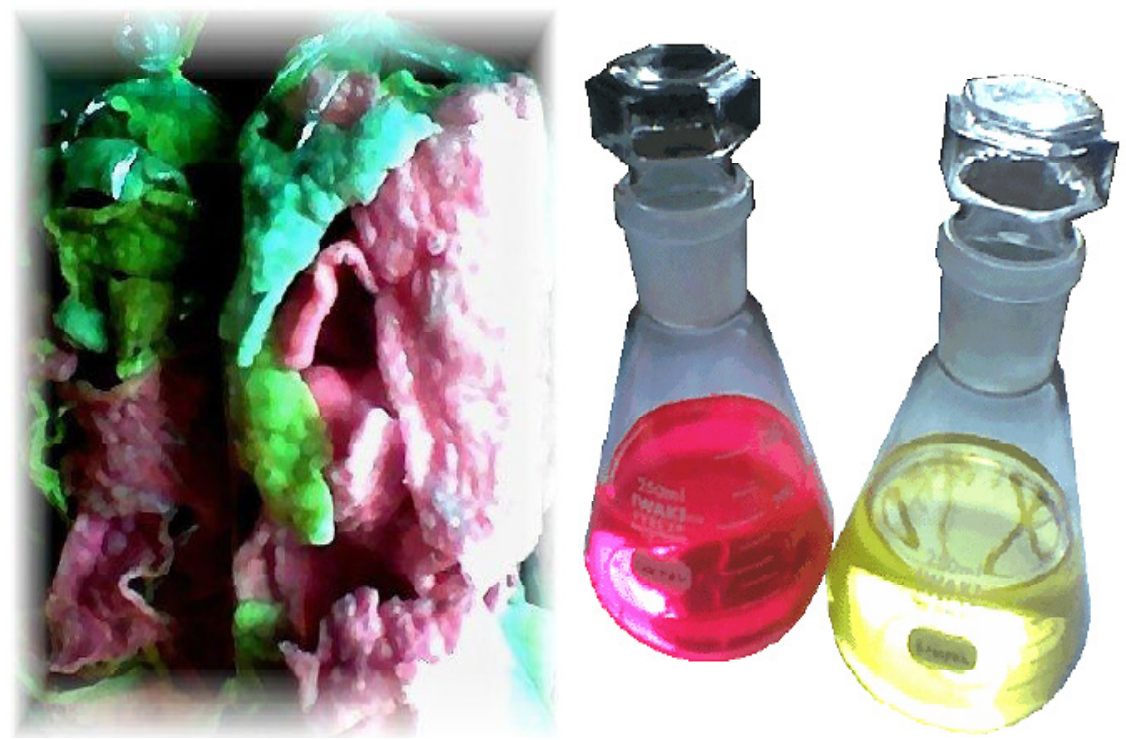

Figure 2. Unregistered colorful crackers (left) and the alkaline ethanolic extracts (right) 


\section{Materials and Methods}

\subsection{Instruments}

Infrared spectrophotometer FTIR-8400 (Shimadzu) and ultraviolet-visible spectrophotometer Specord 200 (AnalitikJena).

\subsection{Chemicals}

Ethanol technical grade $95 \%$ (Bratachem-Indonesia), ammonia analytical grade (E. Merck), isopropanol analytical grade (E. Merck), red (rhodamine B) and yellow dyes (tartrazine) were obtained from National Center of Food and Drug Examination (Pusat Pemeriksaan Obat dan Makanan Nasional), potassium bromate analytical grade (E. Merck), potassium bromide analytical grade (E. Merck), hydrochloric acid 37\% analytical grade (E. Merck), and distilled water.

\subsection{Extraction of the Dyes}

The red and yellow crackers (Figure 2), that were collected from traditional market in Jatinangor, west Java, Indonesia, were ground, weighed and soaked in alkaline ethanolic solvent ( $2 \%$ of ammonia in ethanol), and filtered by using Whatman filter paper. The solvents were evaporated and the dry extracts were ready to be analyzed further.

\subsection{Thin Layer Chromatography}

The viscous extracts were developed on GF254 silica gel plate with a mixture of isopropanol-ammonia (4:0.5) as eluent. The Rf values were compared with those of rhodamine B and tartrazine.

\subsection{Infrared Spectroscopy}

1-2 $\mathrm{mg}$ of dried extracts were dispersed in 300-400 $\mathrm{mg}$ of potassium bromide and were analyzed by using infrared spectrophotometer FTIR-8400 (Shimadzu).

\subsection{Photometric Titration}

The extracts were dissolved in distilled water in volumetric flasks and were pipetted each aliquot $10 \mathrm{~mL}$ into seven titration flasks. Into each flask was added, respectively, $100 \mathrm{mg}$ of potassium bromide and $1 \mathrm{~mL} \mathrm{HCl} 4 \mathrm{~N}$, and dropwise of increasing volumes of $0.01 \mathrm{~N}$ potassium bromate as followed: $0 ; 0.2 ; 0.4 ; 0.6 ; 0.8 ; 1.0$ and 1.2 $\mathrm{mL}$ for red dye sample; and $0 ; 0.15 ; 0.3 ; 0.45 ; 0.6 ; 0.75$; and $0.9 \mathrm{~mL}$ for yellow dye sample.

The absorbance of each mixture solution was measured at its $\lambda_{\max }(551 \mathrm{~nm}$ for red dye and $432 \mathrm{~nm}$ for yellow dye) followed by plotting the absorbance values $v s$ the volume of titrant to obtain the photometric titration curve.

\section{Results and Discussion}

Thin layer chromatograms showed that the dyes were not either rhodamine $\mathrm{B}$ or tartrazine ( $\mathrm{Rf}$ of the red and yellow dyes were 0.714 and 0.652 , respectively, while those of rhodamine B and tartrazine were 0.591 and 0.673 ). These Rf values were preliminary data which confirmed that rhodamine B and tartrazine (both prohibited food dyes) were not contained in both crackers.

Infrared spectroscopy showed characteristic peaks of the red dye (Figure 3) at $3390.6 \mathrm{~cm}^{-1}$ (O-H stretch), 2927.7 $\mathrm{cm}^{-1}$ (C-H stretch of alkyl), $1404.1 \mathrm{~cm}^{-1}$ (O-H bend), $1616.2 \mathrm{~cm}^{-1}$ (C=C stretch), and $1315 \mathrm{~cm}^{-1}$ (C-O stretch). The peaks of yellow dye (Figure 4) occured at $3413.8 \mathrm{~cm}^{-1}$, (O-H stretch), $3213.2 \mathrm{~cm}^{-1}$ (C-H stretch of alkene), $2927.7 \mathrm{~cm}^{-1}$ (C-H stretch of alkyl), $1400.2 \mathrm{~cm}^{-1}$ (O-H bend), $1624.0 \mathrm{~cm}^{-1}$ (C=C stretch) (Silverstein et al., 1991; Lambert et al., 1998). 


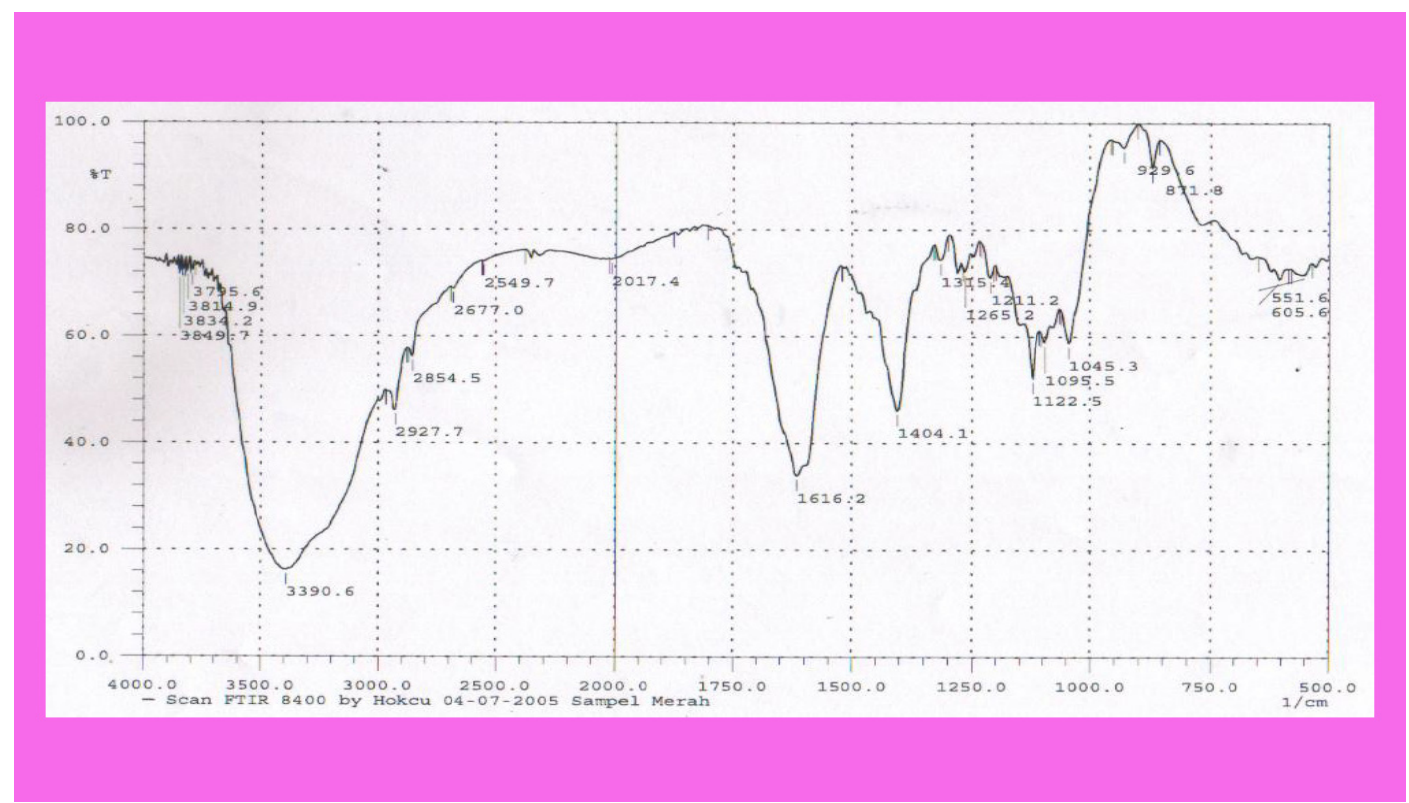

Figure 3. Infrared spectra of the red dye dispersed in potassium bromide

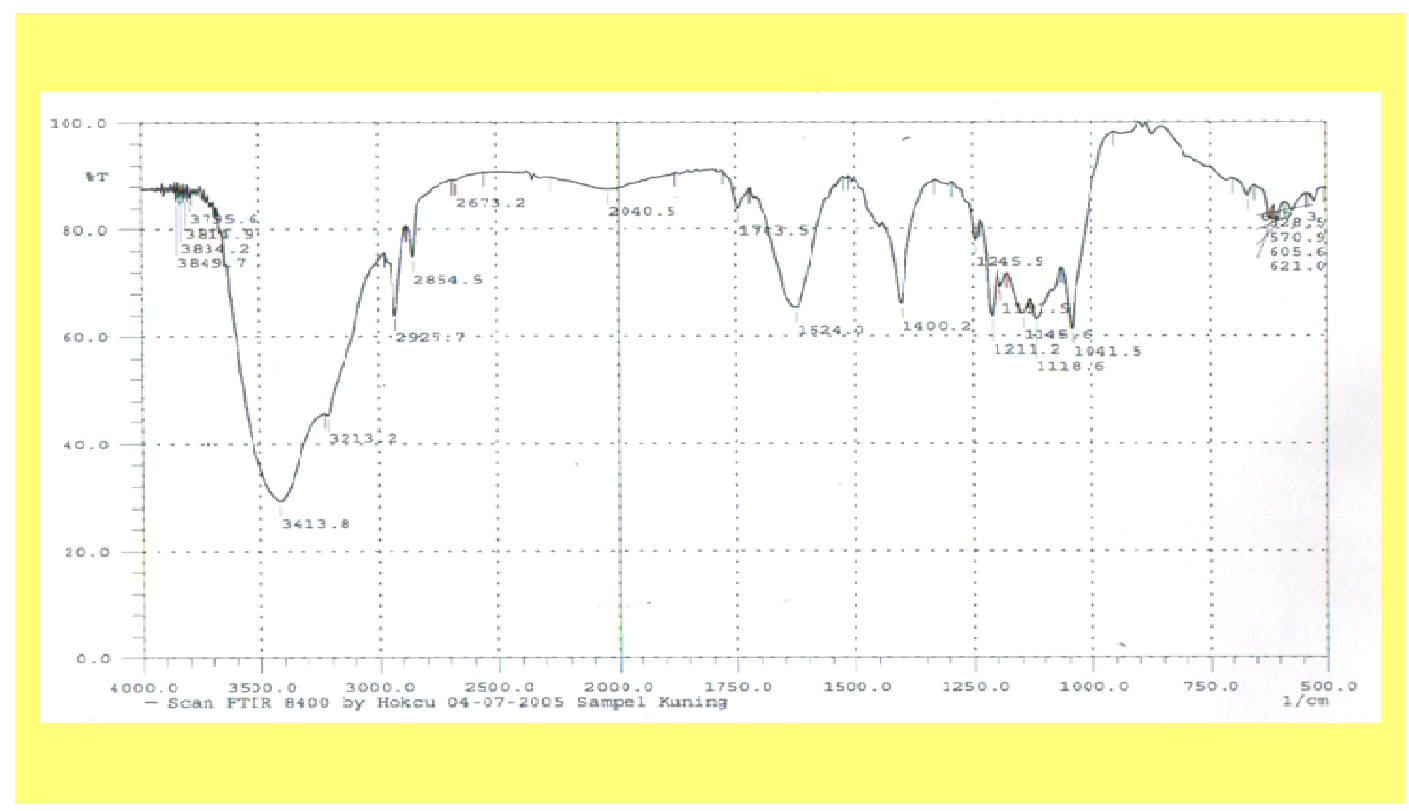

Figure 4. Infrared spectra of the yellow dye dispersed in potassium bromide

The infrared spectrum showed that both red and yellow dyes contained double bond (as showed by $\mathrm{C}=\mathrm{C}$ stretch peaks at about $\left.1600 \mathrm{~cm}^{-1}\right)$. These double bonds can be fruitful targets for bromination reaction.

Visible spectrum (Figure 5 and 6, left) showed $\lambda_{\max }$ at $551 \mathrm{~nm}$ (red dye) and $432 \mathrm{~nm}$ (yellow dye). The measurements of absorbances of photometric titrations were performed at these wavelengths. Compared with the work of Sigmann and Wheeler who focused on the development of a simple spectrophotometric method to determine the quantity of FD\&C color additives present in powdered drink, the $\lambda_{\max }$ of FD\&C Red 40 dye was $503 \mathrm{~nm}$ while that of FD\&C Yellow5 dye was $427 \mathrm{~nm}$ (Sigmann \& Wheeler, 2004). 

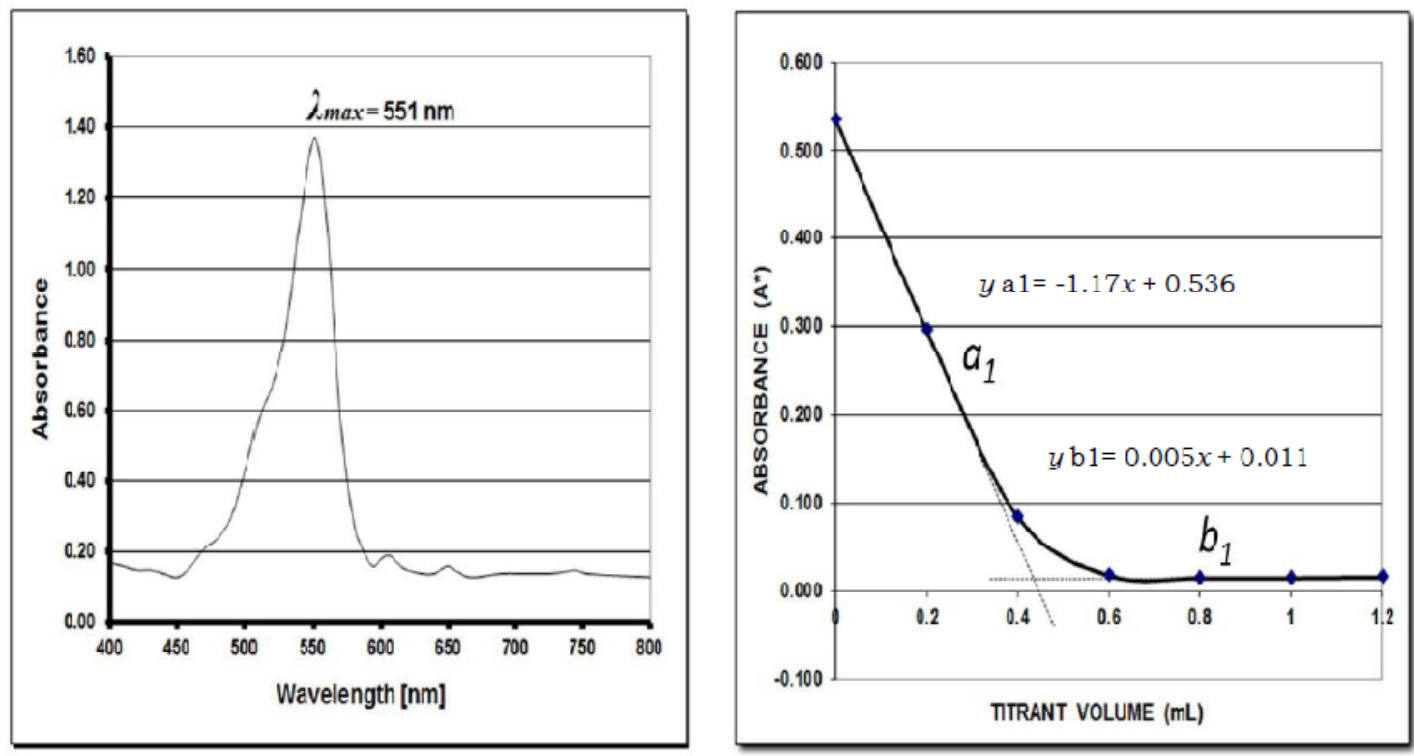

Figure 5. Visible spectrum of red dye (left) and its photometric titration curve (right)
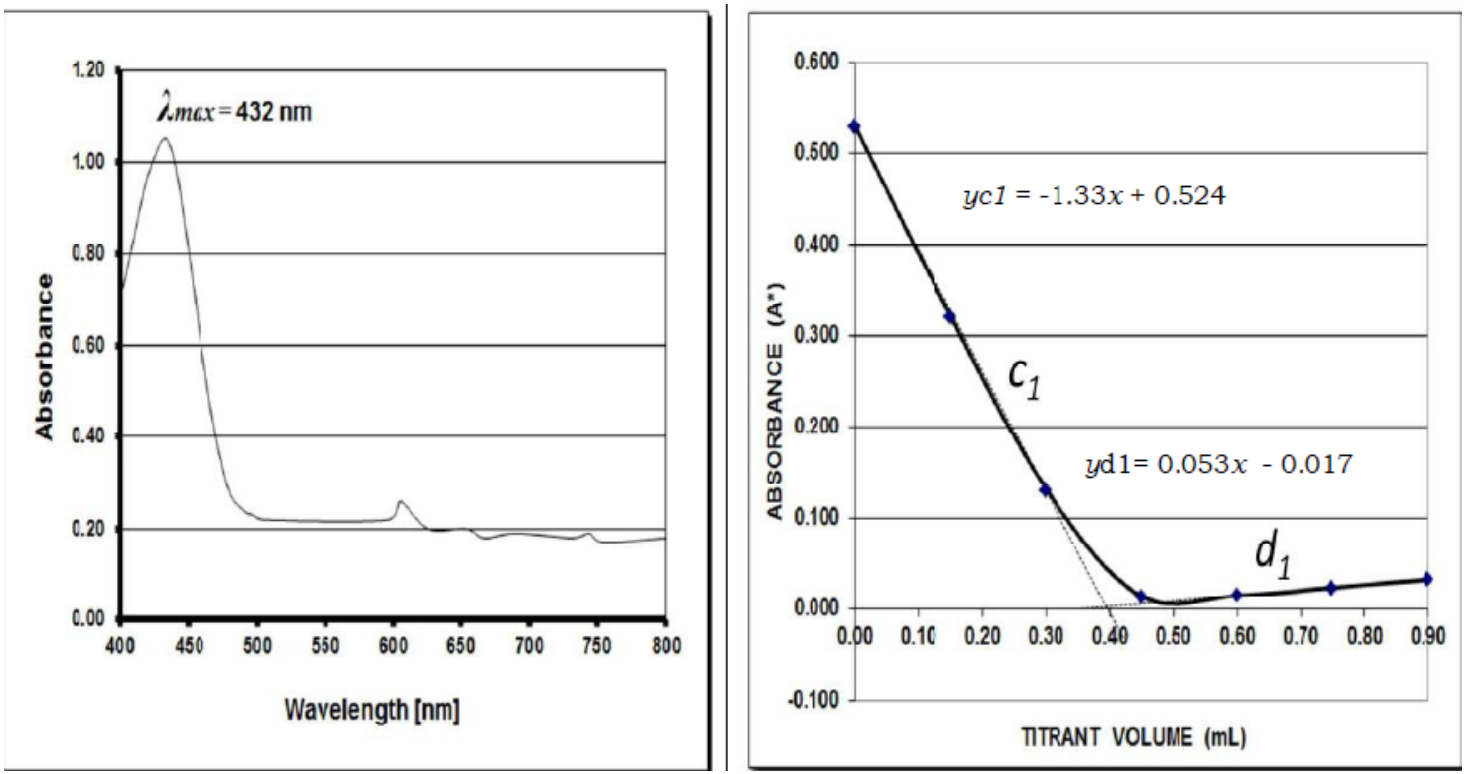

Figure 6. Visible spectrum of yellow dye (left) and its photometric titration curve (right)

Bromine, that was produced in situ by reacting potassium bromide and potassium bromate in acid medium, would attack the double bond via electrophilic addition reaction. The pi $(\pi)$ electrons of the $\mathrm{C}=\mathrm{C}$ double bond can polarize the $\mathrm{Br}-\mathrm{Br}$ bond to the extent that heterolytic cleavage is possible, with a negative bromide ion being released and a positive bromonium ion acting as an electrophile. Alternatively, the $\mathrm{C}=\mathrm{C}$ double bond can be considered as a nucleophile, that attacks the bromine molecule and pushes out the bromide ion, which is a good leaving group.

This type of addition, by means addition of bromine to an alkene, is a very general reaction. As halogenation involves electrophilic attack, substituents on the double bond that increase electron density increase the rate of reaction, whereas electron withdrawing group substituents have the opposite effect (Carey \& Sundberg, 2007). An interesting example of bromination was the work of George and her colleagues. They determined hydrazine by using bromine and methyl red, spectrophotometrically. Their observation suggested ring substitution in the dye molecule with bromine causing steric inhibition of resonance, which was responsible for the decrease in absorbance at $515 \mathrm{~nm}$ and caused decolourisation of the dye (George et al., 2007). 
The intersects of the curves (Figure 5 and 6, right), which were $x_{1}=0.4468$ for red dye and $x_{2}=0.3912$ for yellow dye, indicated the end points of the titration. Each measurements were repeated six times to calculate the precision of the method, and resulted: $\mathrm{SD}=0.0070$ and $\mathrm{CV}=1.60 \%$ for red dye; $\mathrm{SD}=0.0044$ and $\mathrm{CV}=1.13 \%$ for yellow dye. These data confirmed that this photometric titration method was repeatable and fulfilled precision criteria (accuracy was not performed in this work due to limitation of the reference standards).

\section{Conclusion}

Photometric titration method based on the bromination reaction of the dyes showed a decreasing in absorbance due to the conversion of colored analytes to colorless products. This fact indicated that bromination reaction was occured in the red and yellow dyes molecules via electrophilic addition reaction on its double bond, therefore this method is applicable to be used in routine analysis to control illegal food dyes often found in Indonesian home industry crackers.

\section{References}

Carey, F. A., \& Sundberg, R. J. (2007). Advanced Organic Chemistry Part B: Reactions and Synthesis (5th ed.). New York: Springer Science+Business Media, LLC.

George, M., Nagaraja, K. S., \& Balasubramanian, N. (2007). Spectrophotometric Determination of Hydrazine Using Bromine and Methyl Red. Indian Journal of Chemistry, 46A, 1621-1624.

Lambert, J. B., Shurvell, H. F., Lightner, D. A., \& Cooks, R. G. (1998). Organic Structural Spectroscopy. New Jersey: Prentice Hall, Inc.

Sigmann, S. B., \& Wheeler, D. E. (2004). The Quantitative Determination of Food Dyes in Powdered Drink Mixes. Journal of Chemical Education, 81(10), 1475-1478. http://dx.doi.org/10.1021/ed081p1475

Silverstein, R. M., Bassler, G. M., \& Morril, T. C. (1991). Spectrometric Identification of Organic Compounds (5th ed.). USA: John Wiley \& Sons, Inc.

Solomons, T. W. G. (1980). Organic Chemistry (2nd ed.). USA: John Wiley \& Sons, Inc.

Willard, H. H., Merrit, L. L., Dean, J. A., \& Settle, F. A. (1988). Instrumental Methods of Analysis (7th ed.). California: Wadsworth Publishing Company. 